\title{
Interference mitigation in an LTE femtocell base station using uplink antenna selection
}

\author{
Josep Soler-Garrido, Magnus Sandell and Woon Hau Chin ${ }^{*}$
}

\begin{abstract}
Antenna selection represents an attractive technique that allows to improve performance of wireless systems due to its simplicity and ease of implementation. Antenna selection is able to provide diversity through the use of multiple antennas while keeping low cost and energy consumption by selecting only a subset of the available elements at any given time, hence requiring a smaller number of RF transmitter and receiver chains. This makes it especially suitable for femtocell or small-cell base stations, typically employed by cellular operators to provide indoor radio coverage in customer premises. This article presents an analysis and validation of antenna selection using a LTE-FDD femtocell prototype. The effects on antenna selection performance of different antenna training schedules and channel variation rates are investigated under realistic propagation conditions. It is observed that antenna selection is able to provide substantial gains in terms of channel quality and reliability in interference-limited scenarios, attesting its suitability for large-scale femtocell deployments.
\end{abstract}

\section{Introduction}

Cellular data traffic volumes are projected to grow at an exponential rate over the next few years, rendering conventional cellular network structures unable to cope with the expected level of demand. With the efficiency of new generation systems already approaching theoretical limits, one of the main solutions envisioned by wireless operators in order to increase overall network capacity lies in a reduction of the average cell size, hence increasing frequency reuse. Small-cell or femtocell base stations deployments are able to increase capacity, and are ideally suited for indoor applications where a large portion of the traffic is generated and signals from traditional macrocell stations are weak. Femtocells are installed directly by the end-users, possibly inside the coverage area of a larger macrostation [1] which is then able to offload some of its traffic to the femto layer. As a result, deployment of femtocell base stations is strategically important to operators.

However, in this new paradigm, interference is prevalent due to the ad-hoc placement of radio equipment, which inevitably leads to overlapping coverage of multiple cells.

*Correspondence: woonhau.chin@toshiba-trel.com

Toshiba Research Europe Limited, 32 Queen Square, Bristol, BS1 4ND, UK
Therefore, interference is expected to be a major limiting factor which needs to be addressed before large-scale femtocell deployments become a reality [2].

Many techniques can be employed either at network, medium-access control (MAC) or physical layer to control or mitigate interference [3]. Radio resource management [4] and multiantenna techniques such as beamforming [5], combining [6] or interference nulling [7] are popular choices for controlling and mitigating interference.

However, the cost and physical dimensions of femtocell base stations present major restrictions on the choice of interference management techniques. Therefore, conventional multiple antenna methods may not represent the best option for femtocells applications. With regard to this, it should be noted that it is not the antenna elements but the associated radio frequency (RF), analog and mixed-signal circuits that substantially increase the cost and energy requirements of the equipment. Therefore, antenna selection techniques are of particular interest in femtocell applications [8], as only a subset of all the available physical antennas are connected via switches to a reduced number of expensive and power-hungry RF chains including amplifiers, mixers, filters and data converters.

Antenna selection techniques are able to increase link reliability through diversity gains at an order equal to the number of available antenna elements. This leads to lower

\section{望 Springer}

(C) 2012 Soler-Garrido et al.; licensee Springer. This is an Open Access article distributed under the terms of the Creative Commons Attribution License (http://creativecommons.org/licenses/by/2.0), which permits unrestricted use, distribution, and reproduction in any medium, provided the original work is properly cited. 
transmit powers, thereby introducing less interference. In practice however, antenna training is required prior to selection, resulting in a latency increase which can lead to suboptimal antenna choice in fast time-varying wireless channels. Practical RF impairments such as signal loss at the switches, or switching delay and misalignment can also affect performance in practical systems. Consequently, it is not immediately apparent that antenna selection will be beneficial in real systems, and experimental evaluation is required in order to verify the benefits of antenna selection in practical wireless systems.

This article first presents a theoretical analysis of antenna selection, followed by a validation through measurements using a long term evolution-frequency division duplexing (LTE-FDD) femtocell prototype. The rest of the article is organized as follows. Section 2 presents a brief review of the antenna selection concept and a summary of its main advantages and disadvantages. This is followed by an analysis of antenna selection in Section 3. The experimental setup is described in Section 4 and results are presented in Section 5. Finally, the article is concluded in Section 6.

\section{Antenna selection}

\subsection{System model}

We consider a single-user wireless system where the transmitter is assumed to be equipped with a single antenna and the receiver is equipped with an $N$-element antenna array. Let us denote the set of antennas as $a_{1}, \ldots, a_{N}$. In an equivalent narrowband baseband model, there is an associated set of observations $y_{1}, \ldots, y_{N}$ and channel coefficients $h_{1}, \ldots, h_{N}$, related through

$$
y_{n}=h_{n} x+v_{n}
$$

where $x$ is the transmitted symbol and $v_{n}$ is the additive noise. For notational simplicity, we assume unit-power symbols, $E\left\{|x|^{2}\right\}=1$, and define the instantaneous per-branch signal-to-noise ratio (SNR) as

$$
\gamma_{n}=\frac{\left|h_{n}\right|^{2} E\left\{|x|^{2}\right\}}{N_{0}}
$$

where $N_{0}=E\left\{\left|v_{n}\right|^{2}\right\}$ is the noise variance. The average SNR (over the channel realisations) is $\bar{\gamma}_{n}=E\left\{\gamma_{n}\right\}$.

If all of the observations and channel coefficients are available at the receiver's digital baseband and assuming additive white Gaussian noise (AWGN) of equal power across all the antennas, a maximal ratio combiner (MRC) produces a received value given by

$$
r_{\mathrm{mrc}}=\sum_{n=1}^{N} h_{n}^{*} y_{n}
$$

which maximizes the resulting SNR. The resulting SNR in MRC combining is equal to the sum of the SNR of the individual branches [9]

$$
\gamma_{\mathrm{mrc}}=\sum_{n=1}^{N} \gamma_{n} \text {. }
$$

In the case of an OFDM based system, combining is performed on a per-subcarrier basis. Alternative combining methods can be applied in certain cases such as in the presence of severe co-channel interference, allowing for much better performance, if the interferer propagation coefficients or their second order statistics are known at the receiver [10].

The main limitation of such combiners is that they require the availability of baseband signals from all the antennas, which in turn requires $N$ sets of receiver RF and analog chains including antenna, downconverter mixer, filters and analog-to-digital converters. Much simpler receiver architecture employs antenna selection whereby the $N$ elements are connected by switches to a reduced number, $L \leq N$, of RF chains. Therefore, a subset of all the available antennas has to be selected for reception. It can be shown that this architecture, despite showing reduced performance, offers the same level of diversity as a full complexity receiver [11]. Several antenna selection strategies are available depending on the antenna configurations and type of detection performed at the receiver [12]. As an example, if a single antenna is used for transmission and MRC combining is applied at the receiver, a sensible approach is to select the antennas which present the highest SNR, or more generally signal-to-noise plus interference ratio (SINR), at any given time. Assuming $\gamma_{1}, \ldots, \gamma_{N}$ is the set of individual SNR values ordered from highest to lowest, and up to $L$ RF chains are available, the resulting SNR for antenna selection is

$$
\gamma_{\mathrm{mrc}}=\sum_{n=1}^{L} \gamma_{n} \text {. }
$$

In an OFDM system, selection is performed in a bulk fashion for all subcarriers. Moreover, while the diversity order achieved is $N$, coding gain can be significantly lower than that of the full complexity MRC case.

\subsection{Antenna training}

Selection of the antennas requires knowledge of the resulting SNR at each of the elements, which must be obtained through antenna training. In preamble-based wireless systems it would be possible to include additional training fields at the beginning of packets in order to train all the available antennas at the receiver [13]. However, this would translate into significant overheads for large numbers of antennas, as well as the need for very accurate synchronization at the receiver. 
The alternative is to use a single field, hence training a single antenna per packet. In this case, no overheads are incurred, but reception from potentially sub-optimal antennas has to be scheduled periodically in order to train the entire set. If this is done too often, antenna selection is effectively not applied for a significant number of packets. On the other hand, if training is not performed frequently enough, antenna selection performance decreases due to out-of-date information.

An example of antenna training for LTE based on the latter method is illustrated in Figure 1. In this case, $L=1$ is assumed, and CSI for a single antenna is obtained from the reference signals in a subframe. In order to train all the antennas, a sweep across all of them is performed periodically every $T$ subframes. The antenna with the highest SNR, denoted as $a_{S}$, is selected for the duration of the training interval. As aforementioned, a limitation of this approach is the fact that the selected antenna can quickly become suboptimal in rapidly changing channels for large values of $T$.

Training for receive antenna selection has been investigated in, e.g., [13], where a similar setup to Figure 1 was studied and some general results on antenna selection were derived. To analyze the effects of outdated channel estimates, a simple correlation model was used in [13] for the $n$th antenna

$$
h_{n}(t+\delta)=\rho_{n}(\delta) h_{n}(t)+\sqrt{1-\left|\rho_{n}(\delta)\right|^{2}} e_{n}(t+\delta)
$$

where $\rho_{n}(\delta)$ is the temporal channel correlation for lag $\delta$ and $e_{n}(t+\delta)$ is the innovation noise. It was shown in [13] that to minimize the symbol-error rate (SER) in narrowband channels, antenna selection should be based on weighted channel estimates. Since bulk selection in an OFDM system is used in this article, the selection criterion of [13] can be modified to

$$
n_{\max }=\arg \max _{n} w_{n} p_{n}
$$

where $p_{n}=\sum_{k}\left|h_{n, k}\right|^{2}$ is the total power on antenna $n$, summed over all subcarriers. The weights $w_{n}$ reflect how old the channel estimates are and the optimal choice is [13]

$$
w_{n}=\frac{\left|\rho_{n}\left(\delta_{n}\right)\right|^{2}}{\alpha\left(1-\left|\rho_{n}\left(\delta_{n}\right)\right|^{2}\right)+\frac{1+\alpha}{\bar{\gamma}}+\frac{1}{\bar{\gamma}^{2}}}
$$

where $\alpha=E_{p} / E_{s}$ is the energy ratio of the pilots and data symbols. Note that different antennas will have different delays $\delta_{n}$ and hence different correlation $\rho_{n}\left(\delta_{n}\right)$. At high SNR, it is possible to simplify the weights to

$$
w_{n} \approx \frac{\left|\rho_{n}\left(\delta_{n}\right)\right|^{2}}{1-\left|\rho_{n}\left(\delta_{n}\right)\right|^{2}} .
$$

Note that this still requires knowledge of the correlations $\rho_{n}\left(\delta_{n}\right)$; since the delays $\delta_{n}$ are known, the correlation can be derived using the fading rate. If this is not known or the estimation of it is too difficult, then equal weights $w_{n}=1$ must be used. To simplify the receiver we use this choice in the evaluation of the system performance. If $L>1 \mathrm{RF}$ chains are available, (7) can be modified to select the $L$ best antennas.

\section{Performance analysis}

\subsection{SNR analysis}

Assuming all available resource blocks (RB) are employed, the reported SNR corresponds to a sum across the entire bandwidth. If the frequency response on subcarrier $k$ and

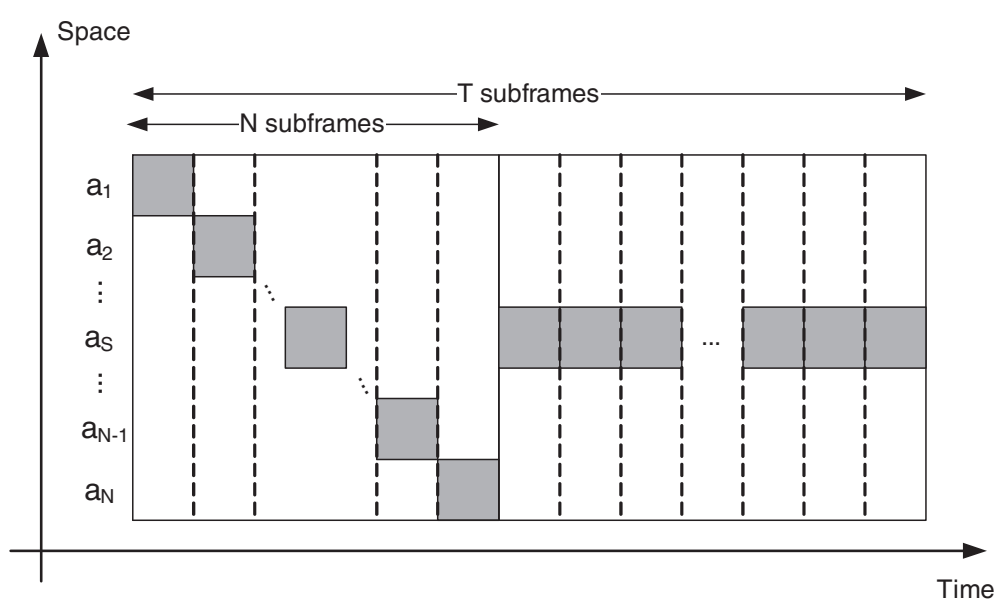

Figure 1 Training schedule where shaded blocks indicate active antennas. 
antenna $n$ is denoted by $h_{n, k}$, we can then relate the time domain channel $\tilde{h}_{n, m}$ through Parseval's theorem

$$
s_{n}=\sum_{k=0}^{K-1} \frac{\left|h_{n, k}\right|^{2}}{N_{0}}=\sum_{m=0}^{M-1} \frac{\left|\tilde{h}_{n, m}\right|^{2}}{N_{0}}=\sum_{m=0}^{M-1} \gamma_{n, m}
$$

where $\gamma_{n, m}$ is the per-tap SINR in the impulse response. For a multipath Rayleigh fading channel where the $M$ taps are independent exponentially distributed variables with respective means $\bar{\gamma}_{m}$, the distribution of the sum can be found as [14]

$$
f_{s_{n}}(x)=\sum_{m=1}^{M} \frac{A_{m}}{\bar{\gamma}_{m}} e^{-x / \bar{\gamma}_{m}}
$$

where

$$
A_{m}=\prod_{m^{\prime} \neq m} \frac{\bar{\gamma}_{m}}{\bar{\gamma}_{m}-\bar{\gamma}_{m^{\prime}}}
$$

are coefficients from a partial fraction expansion.

When antenna selection is applied, the best antenna out of $N$ is used, $s=\max _{n} s_{n}$, whose probability density function (PDF) can then be found as

$$
\begin{aligned}
f_{s}(x) & =N\left(F_{S_{n}}(x)\right)^{N-1} f_{s_{n}}(x) \\
& =N\left(\sum_{m=1}^{M} A_{m} e^{-x / \bar{\gamma}_{m}}\right)^{N-1} \sum_{m=1}^{M} \frac{A_{m}}{\bar{\gamma}_{m}} e^{-x / \bar{\gamma}_{m}}
\end{aligned}
$$

where $F_{s_{n}}(x)$ is the cumulative distribution function (CDF) of $s_{n}$.

\subsection{Error-rate analysis}

To analyze the performance benefits of antenna selection in our setup, we assume for simplicity that perfect channel estimation is available at the receiver and that the channels are independent and flat Rayleigh fading. The diversity order and coding gain are defined as [15]

$$
\begin{aligned}
G_{d} & =\lim _{\bar{\gamma} \rightarrow \infty} \frac{\log P_{b}(\bar{\gamma})}{\log \bar{\gamma}} \\
G_{c} & =\lim _{\bar{\gamma} \rightarrow \infty} \frac{\left(P_{b}(\bar{\gamma})\right)^{-1 / G_{d}}}{\bar{\gamma}} .
\end{aligned}
$$

The asymptotic bit error rate (BER) at high SNR can then be written [16]

$$
P_{b} \approx\left(G_{c} \bar{\gamma}\right)^{-G_{d}} .
$$

In order to deal with generalized selection combining and the proposed selection scheme, we use the analysis technique in [16]. It is based on finding a low-SNR expression of the PDF of the fading SNR (or equivalently using the moment-generating function (MGF)); since the low SNRs dominate the average BER, the asymptotic error rate can be found from this. If the instantaneous BER is $Q(\sqrt{\beta \bar{\gamma}})$, where $\beta$ is the normalized (random) SNR, and the PDF of $\beta$ has the small argument expansion ${ }^{\mathrm{a}}$

$$
f_{\beta}(x)=a x^{t}+o\left(x^{t+\epsilon}\right)
$$

where $0<\epsilon<1$, then the diversity order and coding gain are [16]

$$
\begin{aligned}
G_{d} & =t+1 \\
G_{c} & =\left(\frac{2^{t} a \Gamma(t+3 / 2)}{\sqrt{\pi}(t+1)}\right)^{-1 /(t+1)} .
\end{aligned}
$$

Similarly, if the magnitude of the MGF is

$$
|M(s)|=\left|E\left\{e^{s X}\right\}\right|=b|s|^{-d}+o\left(|s|^{-d}\right), \quad s \rightarrow \infty
$$

then

$$
\begin{aligned}
G_{d} & =d \\
G_{c} & =\left(\frac{2^{d-1} b \Gamma(d+1 / 2)}{\sqrt{\pi} \Gamma(d+1)}\right)^{-1 / d} .
\end{aligned}
$$

In the first phase (see Figure 1), the antennas are swept in $N / L$ subframes to obtain information about all antennas. Since no selection is performed at this stage, the error rate is that of an $L$-branch MRC receiver. The SNR in this case is a $\chi^{2}$-distributed variable with $2 L$ degrees of freedom with the PDF

$$
f_{1}(x)=\frac{x^{L-1} e^{-x}}{(L-1) !}=\frac{1}{(L-1) !} x^{L-1}+o\left(x^{L-1+\epsilon}\right) .
$$

Using (17), the coding gain and diversity order are

$$
\begin{aligned}
& G_{d}^{(1)}=L \\
& G_{c}^{(1)}=\left(\frac{(2 L-1) ! !}{2 L !}\right)^{-1 / L} .
\end{aligned}
$$

This means that $L$ th order diversity is achieved during the first phase.

For the second phase (see Figure 1), the best $L$ antennas are used for reception with maximal ratio combining (MRC). This is known as generalized selection combining (or hybrid selection/MRC) [17] and the MGF has been derived in [18]

$$
\begin{gathered}
M(s)=(1-s)^{-1} \prod_{l=L+1}^{N}\left(1-\frac{s L}{l}\right)^{-1} \\
\Rightarrow|M(s)|=\frac{N !}{L^{N-L} L !} s^{-N}+o\left(|s|^{-N}\right) .
\end{gathered}
$$

Using (19), the coding gain and diversity order are

$$
\begin{aligned}
& G_{d}^{(2)}=N \\
& G_{c}^{(2)}=\left(\frac{(2 N-1) ! !}{2 L^{N-L} L !}\right)^{-1 / N} .
\end{aligned}
$$

Hence $N$ th order diversity is achieved during the second phase. 
When considering the overall BER in the proposed scheme, the average of the two phase should be computed

$$
\begin{aligned}
P_{b} & \approx \frac{N / L}{T}\left(G_{c}^{(1)} \bar{\gamma}\right)^{-G_{d}^{(1)}}+\frac{T-N / L}{T}\left(G_{c}^{(2)} \bar{\gamma}\right)^{-G_{d}^{(2)}} \\
& =\frac{N(2 L-1) ! !}{2 L T L !} \bar{\gamma}^{-L}+\frac{(L T-N)(2 N-1) ! !}{2 T L^{N-L+1} L !} \bar{\gamma}^{-N}(25)
\end{aligned}
$$

It is clear that it only offers $L$ th order diversity since it is limited by that of the first phase (antenna sweeping). However it has a superior coding gain compared to a system without antenna selection; in fact the ratio between them is

$$
\frac{\left(\frac{N(2 L-1) ! !}{2 L T L !}\right)^{-1 / L}}{\left(\frac{(2 L-1) ! !}{2 L !}\right)^{-1 / L}}=\left(\frac{L T}{N}\right)^{1 / L}
$$

If $L=1$, then the ratio is $T / N$; intuitively this makes sense since only a fraction $N / T$ is transmitted without antenna selection and at high SNR it will dominate the BER. The rest of the data is transmitted with antenna selection and will hardly contribute to the overall BER, hence it can be expected that the total BER is $T / N$ times lower than transmitting without antenna selection. For more branches, $L>1$, the improvements are lower since the data transmission without antenna selection is already enjoying diversity through the MRC.

To verify the analysis, the BER of an $L$-branch MRC receiver is shown in Figure 2. The system uses QPSK modulation on independent flat Rayleigh fading channels. The coding gain and diversity from (21) are compared with the asymptotic expression in (15). As can be seen the agreement is very good.

\section{Experimental setup}

As discussed in the previous sections, antenna selection is in theory a cost-effective technique able to provide diversity gain in wireless systems. However, RF impairments and real-life propagation conditions can have a substantial effect in the actual gains observed in practical cases. In this section, we discuss some practical considerations related to antenna selection and describe an experimental setup which is employed to verify performance in an LTE femtocell system.

The first consideration is related to the switching devices required to implement antenna selection. Practical RF switches have a non-negligible insertion loss and switching delay which can potentially negate any antenna selection benefits. Insertion loss typically depends on the operating frequency and the type of device, with solidstate based switches presenting higher losses than for example MEMS-based ones. On the other hand, solidstate devices are typically much faster. Selection of a particular technology has to take into account the target system. In wireless LAN for instance, where channel resources are shared in time between many clients and data is typically transmitted in bursts, switching could be performed during idle time, allowing for low-loss MEMS devices to be employed.

In our case however, given the continuous nature of the LTE signal, switching should occur instantaneously at the start of a subframe so that no data is lost or

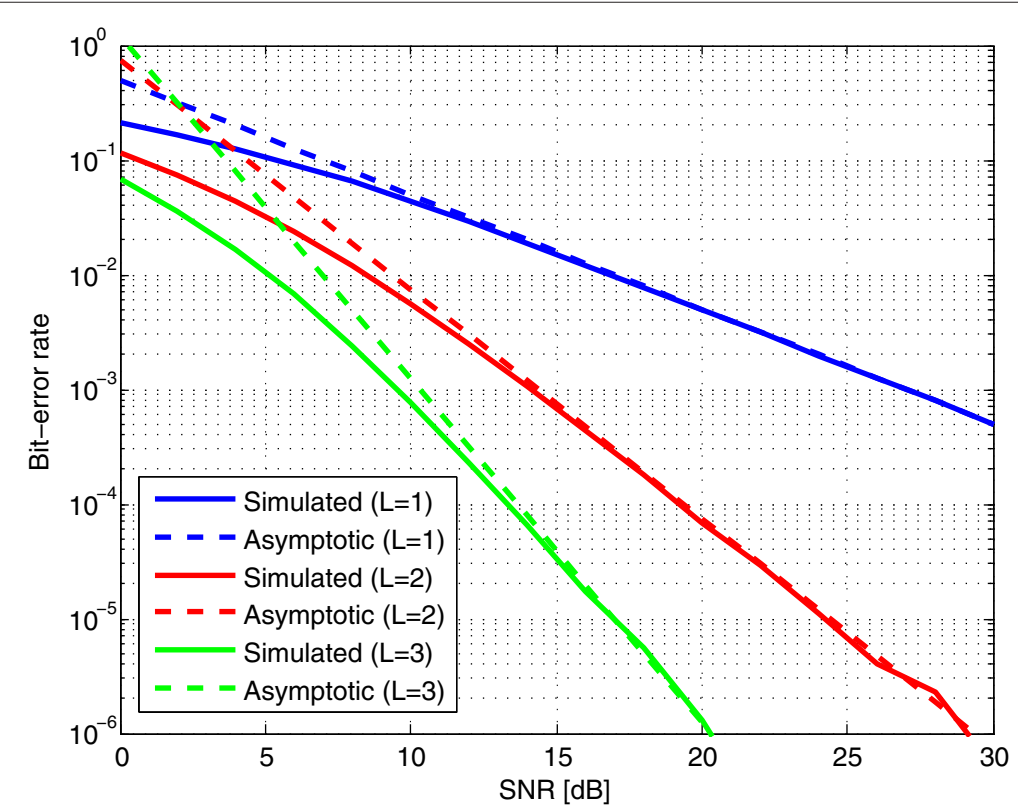

Figure 2 Comparison between the simulated and asymptotic BER for an $L$-branch MRC receiver. 
received through an unintended antenna. This requires fast switches and accurate synchronization of the switching point to the subframe clock in order to prevent any significant loss of data. Synchronization can be particularly difficult in FDD systems, if delay imbalances between uplink and downlink RF and analog circuitry are significant. It would be possible to use two independent sets of antennas and switches for transmission and reception, allowing for independent uplink and downlink optimization. However, this is rarely a cost effective solution and the same antennas are normally used for UL and DL in practice. Therefore, it is important to minimize the delay imbalance between the transmitter and receiver parts of the RF subsystem so that an adequate switching point can be found. Finally, in addition to RF impairments, channel variations in time result in imperfect antenna training which has a negative effect on performance. Therefore, the benefits of antenna selection can only be ultimately validated through prototyping and field measurements performed under realistic propagation conditions.

In this article, we present empirical evaluation of an LTE-FDD femtocell access point employing uplink antenna selection with $N=4$ antenna elements. The setup consists of a picoChip LTE home eNode B (HeNB) development prototype, a Propsim C8 hardware channel emulator and an Aeroflex TM500 LTE test user equipment (UE) and is shown in Figure 3. The HeNB implements a 3GPP LTE Rel. 8 [19] reference design and is limited to a single transmit and receive RF chain. It operates in band 7 at an uplink centre frequency of $2.55 \mathrm{GHz}$ and a bandwidth of $10 \mathrm{MHz}$. The evaluation results presented in the next section are obtained for an uplink configuration where shared channel allocation employs all 50 available RB using DCI (downlink control information) format 0 and MCS (modulation coding scheme) values ranging from 0 to 15 and 19 , hence covering different modulation formats up to 16 QAM.
The femtocell prototype is complemented by an antenna switching setup consisting of an external FPGA board and a set of Minicircuits ZFSWA2-63DR + single-pole doublethrow (SPDT) switches. The switches have a 35 ns switching time, a $1.4 \mathrm{~dB}$ insertion loss each and a bandwidth from $500 \mathrm{MHz}$ up to $6 \mathrm{GHz}$.

Therefore, the expected insertion loss in our system, corresponding to the serial connection of two switches, is $2.8 \mathrm{~dB}$. It should be noted that suitable devices with lower insertion loss are available. With regard to synchronization, switching delay effects are expected to be negligible as the selected devices perform switching in approximately 1 time-domain sample for the LTE system where, given a maximum number of subcarriers of 2048 with $15 \mathrm{kHz}$ separation, the sampling time is $T_{s}=$ $1 /\left(15 \cdot 10^{3} \times 2048\right) \mathrm{s} \approx 32.5 \mathrm{~ns}$. In a similar way, the effect of uplink/downlink imbalance, in the order of a few tens of samples, is well within the approximately $5 \mu \mathrm{s}$ OFDM cyclic prefix, making it relatively simple to find an appropriate switching point.

The desired propagation conditions are implemented by a PropSim C8 channel emulator. Due to the indoor nature of femtocell systems and the similar operating frequencies, TGn channel models typically employed in IEEE 802.11n wireless LAN simulations are used [20]. Delay profiles and antenna correlation values are programmed into the hardware emulator, and a set of impulse responses are generated for the experiments. Different mobile terminal speeds are simulated by changing the channel impulse response update rate. An Aeroflex TM500 test UE is employed as mobile terminal. It connects in HARQ mode to the femtocell access point, i.e., communication is only established at physical layer.

Finally, an interferer is added in some of the experiments in order to evaluate the performance of antenna selection in an interference-loaded scenario. In this case, a second uplink LTE source is connected to the channel emulator.

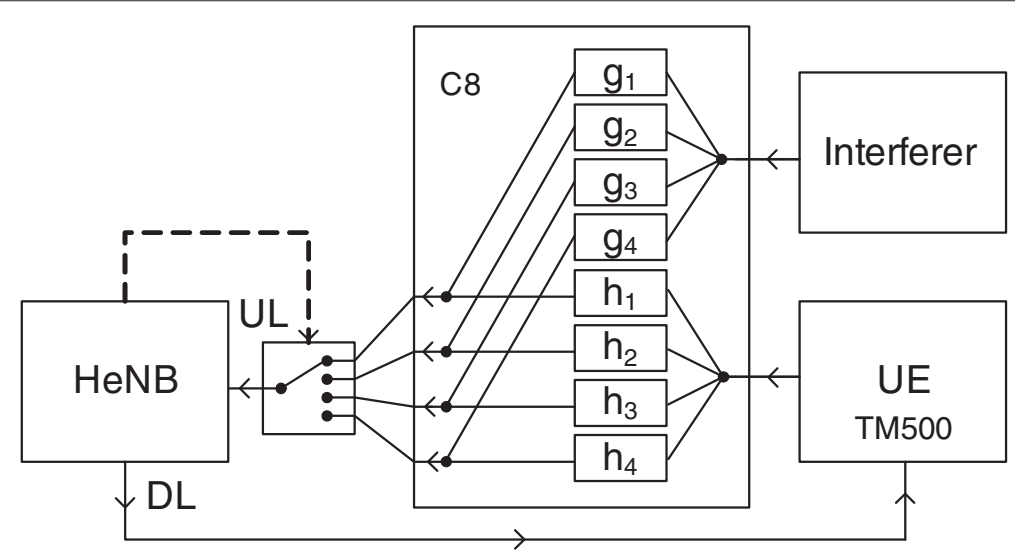

Figure 3 Evaluation setup. 
Table 1 Evaluation parameters for Trial 1

\begin{tabular}{ll}
\hline UL channel models & TGn B, TGn C \\
UE speed & $0.1-2.0 \mathrm{~m} / \mathrm{s}$ \\
UE transmit power & $-10 \mathrm{dBm}$ \\
UL average path loss & $\approx 37.7 \mathrm{~dB}(\mathrm{~B}), 36.2 \mathrm{~dB}(\mathrm{C})$ \\
Number of antennas ( $N)$ & 1 (fixed ant.) or 4 (ant. sel.) \\
Antenna separation & 1 wavelength \\
Antenna training rate $(T)$ & 20 or 100 subframes
\end{tabular}

Two sets of channels are generated in this case, $h_{1}, \ldots, h_{4}$ for the desired user and $g_{1}, \ldots, g_{4}$ for the interferer. The resulting signals after propagation are combined and fed to the femtocell.

The complete setup is depicted in Figure 3, where it can be observed that only uplink propagation is simulated, whereas a direct connection between femtocell and terminal is used for downlink communication. Hence, the effects of imperfect terminal synchronization, for example in cases of weak downlink signal during deep fades, are not a factor in the results presented in this article.

\section{Performance evaluation}

Performance is evaluated in terms of average SINR at the receiver, which is measured in intervals of approximately 1 second at the femtocell base station. Additionally, average cyclic redundancy check (CRC) error rate is recorded at the same rate. We evaluate performance for the case of $N=1$ (fixed antenna) and $N=4, L=1$ (antenna selection) under different propagation scenarios. Three different propagation channels are considered, including a typical residential environment (channel TGn channel B, $15 \mathrm{~ns}$ rms delay spread), a small office environment (TGn channel C, $30 \mathrm{~ns}$ rms delay spread) and a typical office (TGn channel D, $50 \mathrm{~ns}$ rms delay spread). A linear array of omnidirectional antennas with a separation of one wavelength is considered. The effect of different UE speeds and antenna training rates are evaluated.

\subsection{Performance in an interference-free scenario}

Initially, an interference-free case is considered. In order to keep an identical configuration in both fixed antenna and antenna selection cases, the fixed antenna measurements were obtained by applying a fixed control value to the switches. In a practical case however, the insertion loss of the switches would not be present in the fixed antenna case, so it has to be subtracted from any observed gains from antenna selection. The main parameters for Trial 1 are summarized in Table 1 . Figures 4 and 5 show the empirical PDF of the SINR measurements at the receiver for channel $\mathrm{C}$ and UE speeds of 0.1 and $1.0 \mathrm{~m} / \mathrm{s}$ using $T=100$ and $T=20$ subframes, respectively, where each subframe is $1 \mathrm{~ms}$ long.

Similar results for channel B can be found in [21]. As expected, antenna selection increases the average measured SINR, and also reduces its variance, resulting in a more reliable link. This effect is more noticeable in the case of a slowly changing channel as the optimal antenna is likely to remain the same for the duration of the trainingselection interval. In this case it is possible to compare the experimental data with the theoretical one for the channel

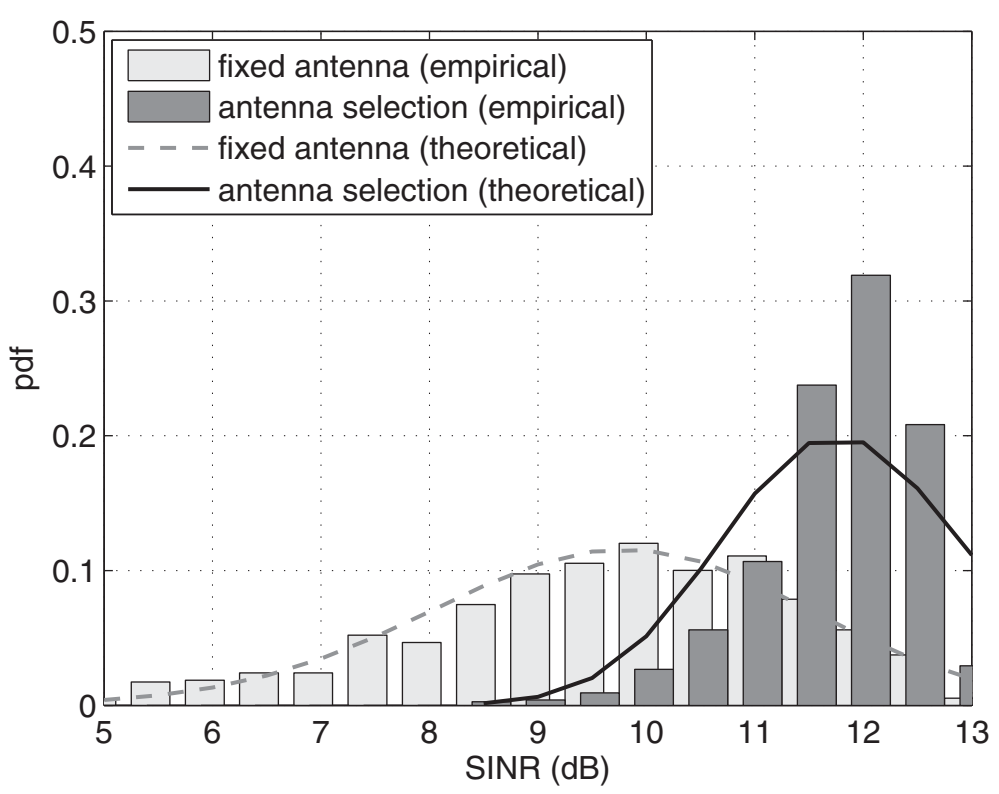

Figure 4 Empirical and theoretical SINR distributions for channel C with UE speed of $0.1 \mathrm{~m} / \mathrm{s}$ and $T=100$. 


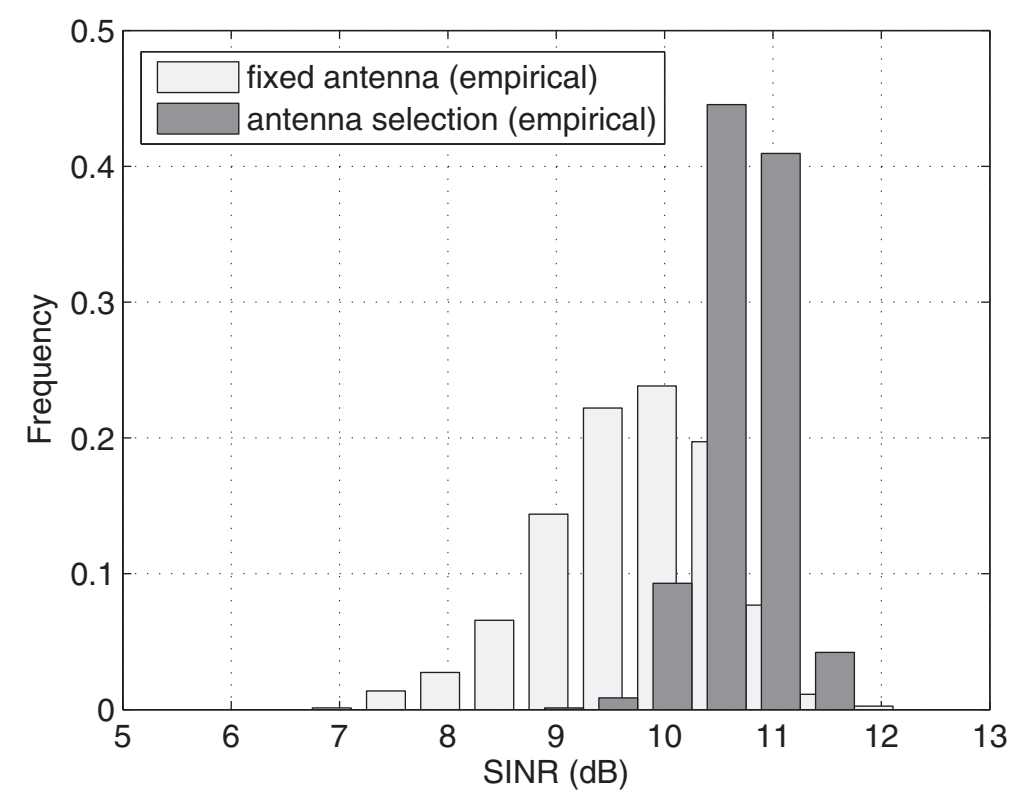

Figure 5 Empirical SINR distributions for channel C with UE speed of $1 \mathrm{~m} / \mathrm{s}$ and $T=20$.

model after adjustments to match the average receiver SINR and the measuring capabilities of the system, which provides estimates in steps of $0.5 \mathrm{~dB}$. This comparison is made for the case of $0.1 \mathrm{~m} / \mathrm{s}$ UE speed where the averaging effects during the measurement intervals are smaller. In that case it is observed that experimental and theoretical data agree to a large extent. The average SINR measured at the receiver increases from 9.9 to $11.8 \mathrm{~dB}$, i.e., a gain of $1.9 \mathrm{~dB}$ is observed.
In the case of faster update rate, a reduced standard deviation is observed, but it is only a result of averaging over a larger number of channel impulse responses during the $1 \mathrm{~s}$ measurement period. In any case, antenna selection performance is degraded, and the difference with the fixed antenna case is reduced as faster channel variations result in more frequent suboptimal antenna selection.

Figure 6 shows average SINR values for all the experiments in Trial 1 using channels B and C. Similar gains
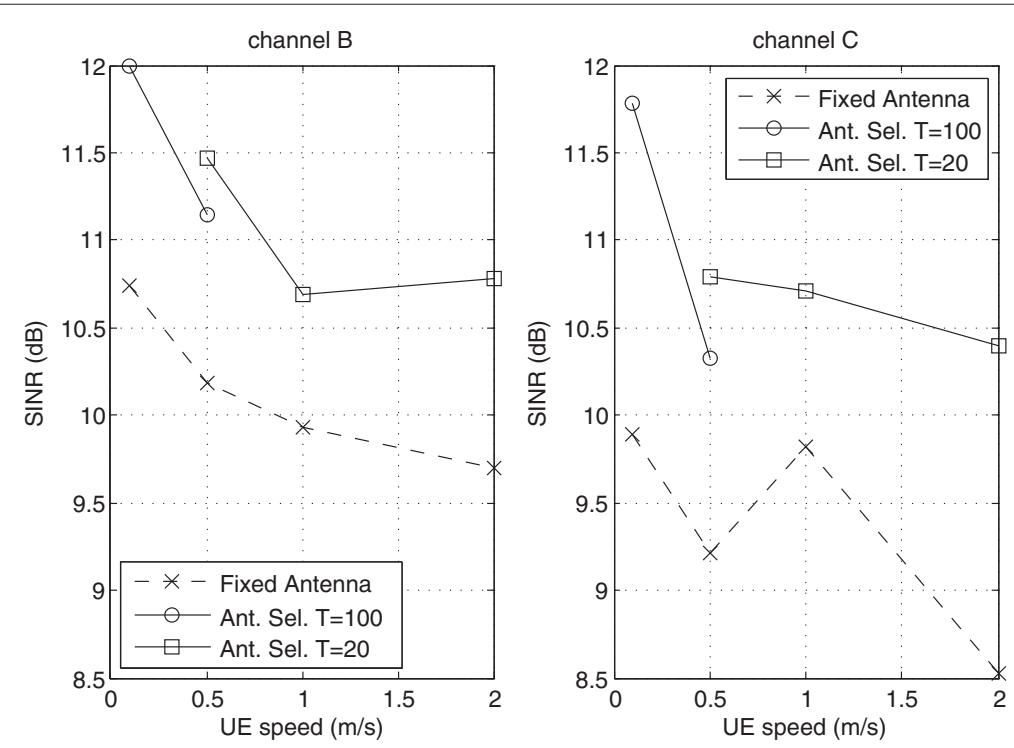

Figure 6 Measured average SINR values. 


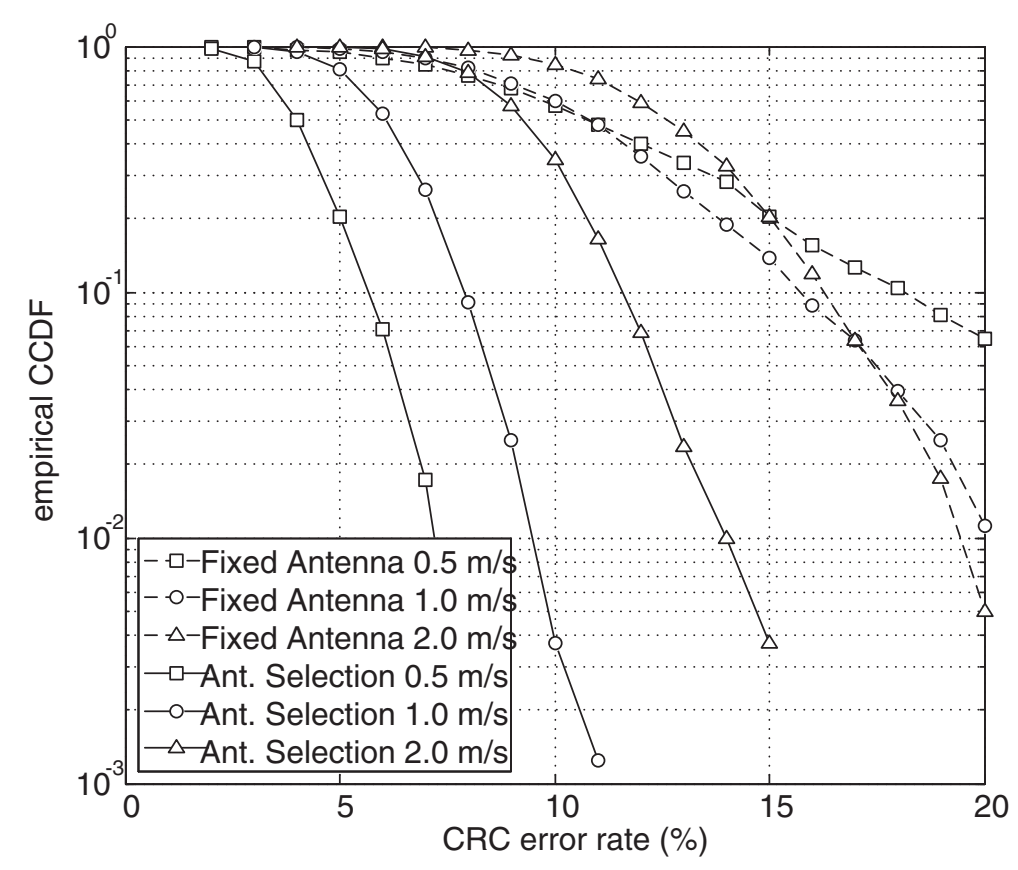

Figure 7 Empirical error rate CCDF for channel C.

are observed for both channels, so a small increase in frequency selectivity does not substantially degrade antenna selection performance. In general, the degradation is similar for both fixed antenna and antenna selection cases, i.e., performance gains remain largely constant at the speeds of interest, and are typically in the $1-2 \mathrm{~dB}$ range. Hence, the SINR gains observed fail to make up for the insertion loss of the switches in this case.

The increase in reliability for antenna selection system is also apparent in the complementary cumulative density

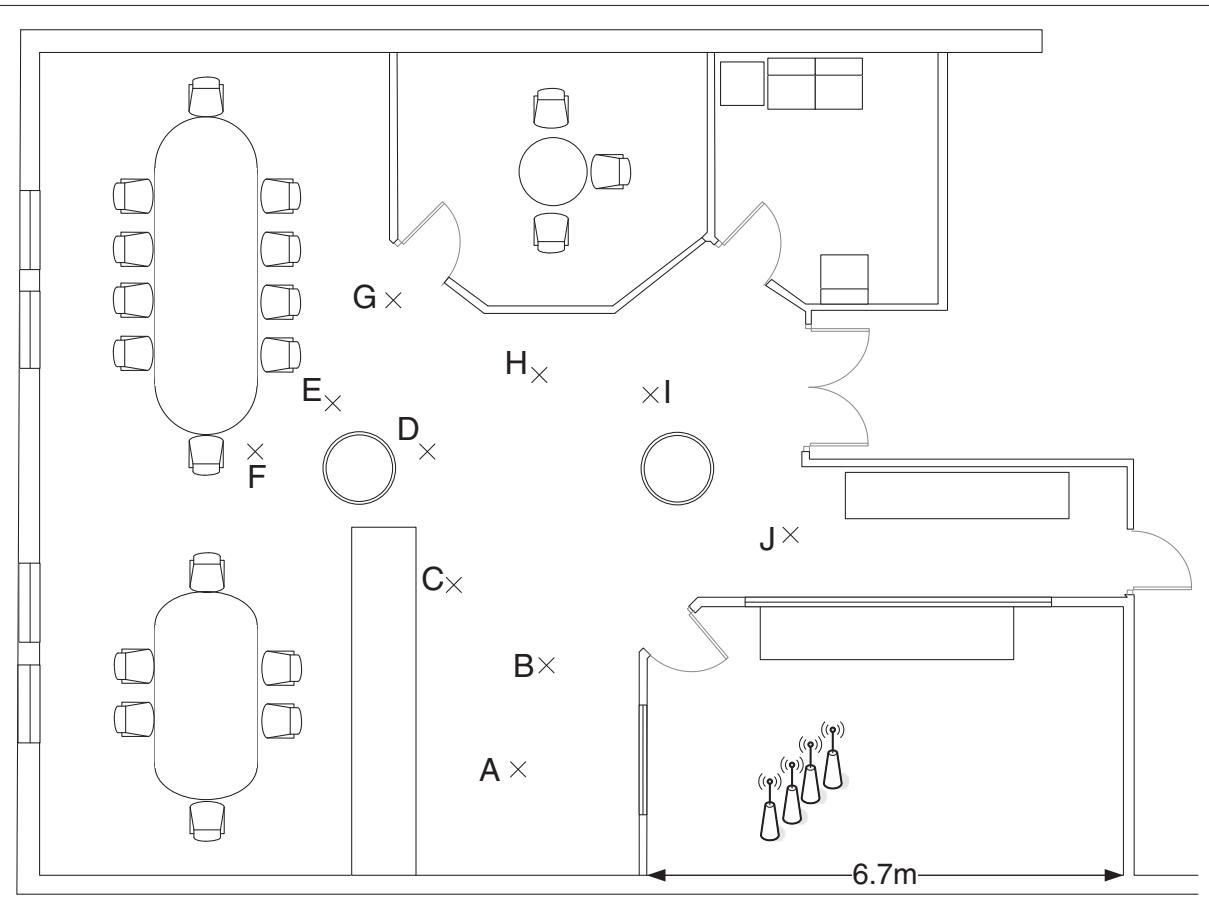

Figure 8 Floor plan with antenna positions during evaluation. 


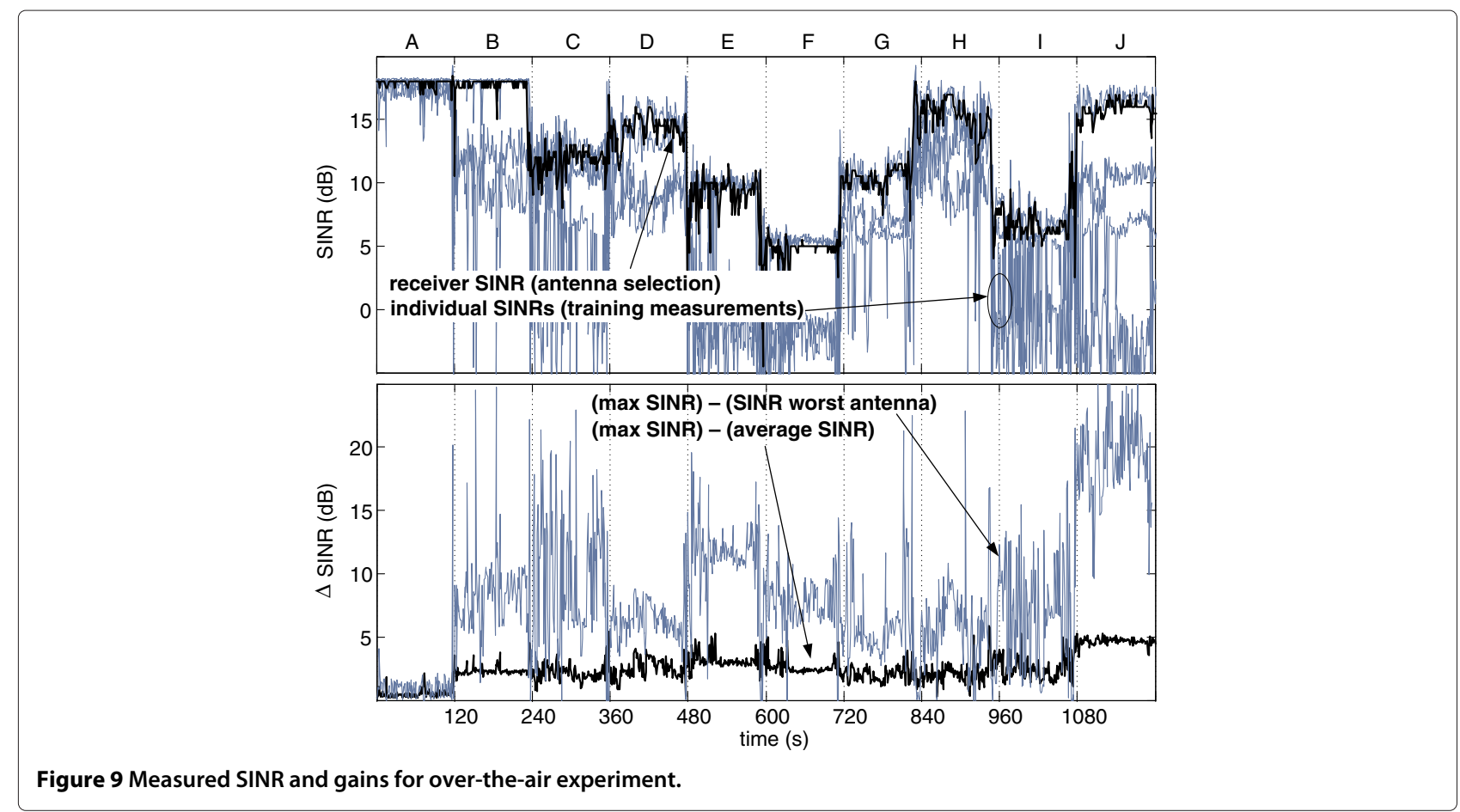

function (CCDF) of the CRC error rate shown in Figure 7, where it can be observed that the probability of finding higher error rates decreases more rapidly when multiple antennas are available.
Cyclic redundancy check results are plotted in Figure 12 and again, substantial gains are measured in terms of average error rates. For instance, the probability of finding error rates higher than $20 \%$ during a measuring interval

Table 2 Summary of over-the-air results

\begin{tabular}{|c|c|c|c|c|c|c|}
\hline Position & Rx SINR & Maximum SINR & Antenna 1 & Antenna 2 & Antenna 3 & Antenna 4 \\
\hline \multirow[t]{2}{*}{ A } & $17.87 \mathrm{~dB}$ & $18.19 \mathrm{~dB}$ & $17.81 \mathrm{~dB}$ & $18.08 \mathrm{~dB}$ & $17.46 \mathrm{~dB}$ & $17.29 \mathrm{~dB}$ \\
\hline & & $(+0.32 \mathrm{~dB})$ & $(-0.06 \mathrm{~dB})$ & $(+0.21 \mathrm{~dB})$ & $(-0.41 \mathrm{~dB})$ & $(-0.58 \mathrm{~dB})$ \\
\hline \multirow[t]{2}{*}{ B } & $17.61 \mathrm{~dB}$ & $18.05 \mathrm{~dB}$ & $10.57 \mathrm{~dB}$ & $18.02 \mathrm{~dB}$ & $17.73 \mathrm{~dB}$ & $12.07 \mathrm{~dB}$ \\
\hline & & $(+0.44 \mathrm{~dB})$ & $(-7.04 \mathrm{~dB})$ & $(+0.41 \mathrm{~dB})$ & $(+0.12 \mathrm{~dB})$ & $(-5.54 \mathrm{~dB})$ \\
\hline \multirow[t]{2}{*}{ C } & $12.03 \mathrm{~dB}$ & $13.23 \mathrm{~dB}$ & $11.22 \mathrm{~dB}$ & $7.55 \mathrm{~dB}$ & $12.68 \mathrm{~dB}$ & $10.81 \mathrm{~dB}$ \\
\hline & & $(+1.20 \mathrm{~dB})$ & $(-0.81 \mathrm{~dB})$ & $(-4.48 \mathrm{~dB})$ & $(+0.65 \mathrm{~dB})$ & $(-1.22 \mathrm{~dB})$ \\
\hline \multirow[t]{2}{*}{$\mathrm{D}$} & $14.33 \mathrm{~dB}$ & $15.15 \mathrm{~dB}$ & $9.95 \mathrm{~dB}$ & $15.08 \mathrm{~dB}$ & $13.62 \mathrm{~dB}$ & $9.07 \mathrm{~dB}$ \\
\hline & & $(+0.82 \mathrm{~dB})$ & $(-4.38 \mathrm{~dB})$ & $(+0.75 \mathrm{~dB})$ & $(-0.71 \mathrm{~dB})$ & $(-5.26 \mathrm{~dB})$ \\
\hline \multirow[t]{2}{*}{$E$} & $9.10 \mathrm{~dB}$ & $9.98 \mathrm{~dB}$ & $9.60 \mathrm{~dB}$ & $9.24 \mathrm{~dB}$ & $-0.17 \mathrm{~dB}$ & $-0.52 \mathrm{~dB}$ \\
\hline & & $(+0.88 \mathrm{~dB})$ & $(+0.50 \mathrm{~dB})$ & $(+0.14 \mathrm{~dB})$ & $(-9.27 \mathrm{~dB})$ & $(-9.26 \mathrm{~dB})$ \\
\hline \multirow[t]{2}{*}{ F } & $5.18 \mathrm{~dB}$ & $6.36 \mathrm{~dB}$ & $5.75 \mathrm{~dB}$ & $5.87 \mathrm{~dB}$ & $-0.37 \mathrm{~dB}$ & $-0.12 \mathrm{~dB}$ \\
\hline & & $(+1.18 \mathrm{~dB})$ & $(+0.57 \mathrm{~dB})$ & $(+0.69 \mathrm{~dB})$ & $(-5.55 \mathrm{~dB})$ & $(-5.30 \mathrm{~dB})$ \\
\hline \multirow[t]{2}{*}{ G } & $11.64 \mathrm{~dB}$ & $12.22 \mathrm{~dB}$ & $11.07 \mathrm{~dB}$ & $12.03 \mathrm{~dB}$ & $8.09 \mathrm{~dB}$ & $8.23 \mathrm{~dB}$ \\
\hline & & $(+0.58 \mathrm{~dB})$ & $(-0.57 \mathrm{~dB})$ & $(+0.39 \mathrm{~dB})$ & $(-3.55 \mathrm{~dB})$ & $(-3.41 \mathrm{~dB})$ \\
\hline \multirow[t]{2}{*}{$\mathrm{H}$} & $14.99 \mathrm{~dB}$ & $15.77 \mathrm{~dB}$ & $12.80 \mathrm{~dB}$ & $15.57 \mathrm{~dB}$ & $14.22 \mathrm{~dB}$ & $10.27 \mathrm{~dB}$ \\
\hline & & $(+0.78 \mathrm{~dB})$ & $(-2.19 \mathrm{~dB})$ & $(+0.58 \mathrm{~dB})$ & $(-0.77 \mathrm{~dB})$ & $(-4.72 \mathrm{~dB})$ \\
\hline \multirow[t]{2}{*}{1} & $7.28 \mathrm{~dB}$ & $8.44 \mathrm{~dB}$ & $3.39 \mathrm{~dB}$ & $4.18 \mathrm{~dB}$ & $6.94 \mathrm{~dB}$ & $7.04 \mathrm{~dB}$ \\
\hline & & $(+1.16 \mathrm{~dB})$ & $(-3.89 \mathrm{~dB})$ & $(-3.10 \mathrm{~dB})$ & $(-0.34 \mathrm{~dB})$ & $(-0.24 \mathrm{~dB})$ \\
\hline \multirow[t]{2}{*}{ J } & $15.85 \mathrm{~dB}$ & $16.60 \mathrm{~dB}$ & $10.37 \mathrm{~dB}$ & $7.12 \mathrm{~dB}$ & $16.60 \mathrm{~dB}$ & $-1.27 \mathrm{~dB}$ \\
\hline & & $(+0.75 \mathrm{~dB})$ & $(-5.48 \mathrm{~dB})$ & $(-8.73 \mathrm{~dB})$ & $(+0.75 \mathrm{~dB})$ & $(-17.12 \mathrm{~dB})$ \\
\hline
\end{tabular}


Table 3 Evaluation parameters for Trial 2 (moderate interference)

\begin{tabular}{ll}
\hline UL UE/INT channel model & TGn B/TGn C \\
UE/INT speed & $0.25 \mathrm{~m} / \mathrm{s}$ both \\
UE/INT transmit power & $-4 /-20 \mathrm{dBm}$ \\
UE/INT average path loss & $\approx 42 / 33.1 \mathrm{~dB}$ \\
Number of antennas (N) & 1 (fixed ant.) or 4 (ant. sel.) \\
Antenna separation & 1 wavelength \\
Antenna training rate $(\mathrm{T})$ & 100 subframes \\
\hline
\end{tabular}

in Trial 2 was around 0.80 for the fixed-antenna case and 0.14 for the antenna-selection case. These results are particularly significant as interference is expected to be one of the major performance limiting factors in femtocell-based cellular systems.

However, larger degradation with increasing user speed is observed in the antenna selection case. In summary, antenna selection is expected to offer limited benefits when insertion loss due to switching is considered in our practical setup with four antenna elements and a single RF chain. Therefore, using an appropriate switching device is critical, and net gains could potentially be achieved with high-end single-pole four-throw (SP4T) switches with low insertion loss.

Finally, even when gains in terms of average SINR are not significant, spatial diversity can be substantially beneficial in practical scenarios where terminals remain static or move at very low speeds. This is frequently the case for laptops and other appliances which are typically used for large periods of time in a fixed location. In that case, it is possible to experience deep fades that severely impair performance, and antenna selection capabilities can dramatically reduce the impact of this. This is illustrated by an actual over-the-air experiment conducted with the femtocell kit in an office environment detailed in the floor plan of Figure 8. In this case, the base station remains fixed in the position indicated by the antenna symbols, and the terminal is situated on ten different positions labeled A-J, staying on each one of them for $2 \mathrm{~min}$ before moving to the next. The training interval for this experiment is set at $T=100$ and omnidirectional antennas with a physical spacing of $15 \mathrm{~cm}$ are employed.

Measurements for this field trial are shown in the top plot of Figure 9, where SINR values obtained during training of each antenna are shown along with the overall SINR for the antenna selection receiver. In this case, a training interval $T=100$ is employed. It is observed how the measured receiver SINR is able to closely track the maximum SINR, and substantial gains from antenna selection can be observed, especially at certain antenna positions. In addition, the antenna selection receiver is able to greatly reduce link quality variance compared to the individual antennas.
The advantages of antenna selection become more obvious in the bottom plot where the difference between the maximum instantaneous SINR and both the worst antenna at each position and the spatial average and are plotted. Gains in the order of 1.5 to $3 \mathrm{~dB}$ are consistently measured against the average for most positions, and differences in excess of 5-10 dB are recorded between the selection receiver and the worst antennas. Table 2 shows the measured SINR for all four antennas in ten different locations averaged over the $120 \mathrm{~s}$ period, along with the average receiver SINR and the average instantaneous maximum SINR across all the antennas. The values in brackets indicate increments with respect to the average receiver SINR. It is observed that the SINR stays approximately within $1 \mathrm{~dB}$ of the maximum SINR. This small difference is expected as the former is an average over a measuring period including training and fixed selection for $T=$ 100 subframes whereas the latter results from averaging instantaneous maximum values at specific subframes.

However, it is the comparison with average SINR for the individual antennas that indicate the potential benefits of antenna selection. As observed, all of the antennas suffer from severe signal losses at several positions. In many cases the degradation is much greater than the insertion loss of the switches. Therefore, net gains are obtained in real-life scenarios, where antenna selection is able to reduce the likelihood of experiencing long-duration deep signal fades in static propagation environments.

\subsection{Performance in the presence of interference}

In an interference-loaded case the insertion loss of the switches is less critical as SINR is largely determined by co-channel interference at the antenna elements. Two additional experiments using an interference source are performed with the parameters listed in Tables 3 and 4, corresponding to moderate and severe interference.

The first case simulates a scenario where an interfering terminal is in the close vicinity of the desired user, but still the average power of the desired signal is several $\mathrm{dB}$ stronger. In the second case the observed propagation channel from both desired and interfering user to the base station are similar in terms of attenuation and delay

Table 4 Evaluation parameters for Trial 3 (severe interference)

\begin{tabular}{ll}
\hline UL UE/INT channel model & TGn C both \\
UE/INT speed & $0.25 \mathrm{~m} / \mathrm{s}$ both \\
UE/INT transmit power & $-4 /-20 \mathrm{dBm}$ \\
UE/INT average path loss & $\approx 39.1 / 22.1 \mathrm{~dB}$ \\
Number of antennas (N) & 1 (fixed ant.) or 4 (ant. sel.) \\
Antenna separation & 1 wavelength \\
Antenna training rate $(\mathrm{T})$ & 100 subframes
\end{tabular}




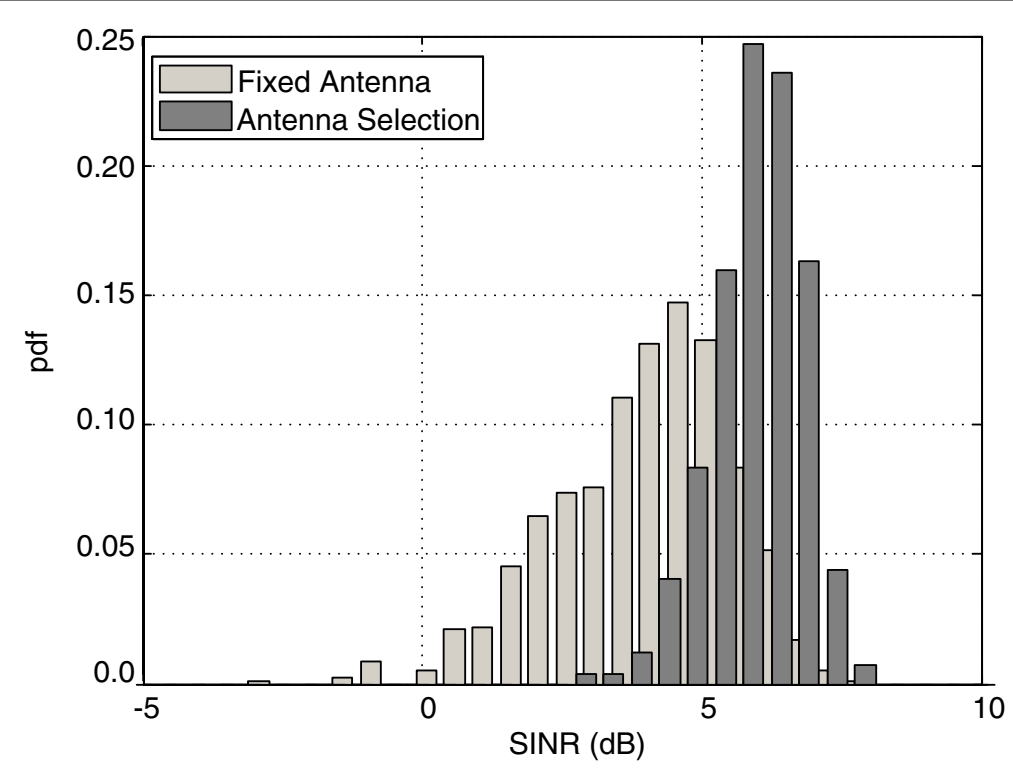

Figure 10 Empirical SINR distributions for Trial 2.

spread. For practical reasons, the transmit power of the interference source during the experiments was set at a level much lower than that of the desired terminal, but this was offset by a reduced average propagation loss. In both trials, both the UE and interferer speeds were set at a low value of $0.25 \mathrm{~m} / \mathrm{s}$ and accordingly a longer antenna training interval $(T=100)$ is selected.

Results in terms of SINR are shown in Figures 10 and 11. As expected, the results show substantially degraded SINR values due to the presence of interference. However, the observed antenna selection gains are higher. For Trial 2, an increase in average SINR from 3.0 to $5.3 \mathrm{~dB}$ is obtained when antenna selection is enabled, whereas for Trial 3 the improvement is from approximately 1.5 to $4.3 \mathrm{~dB}$.

\section{Conclusion}

In this article, the benefits of antenna selection in practical indoor femtocell wireless systems have been analyzed and experimentally validated by means of laboratory and field trials. Gains in terms of increased SINR and reduced

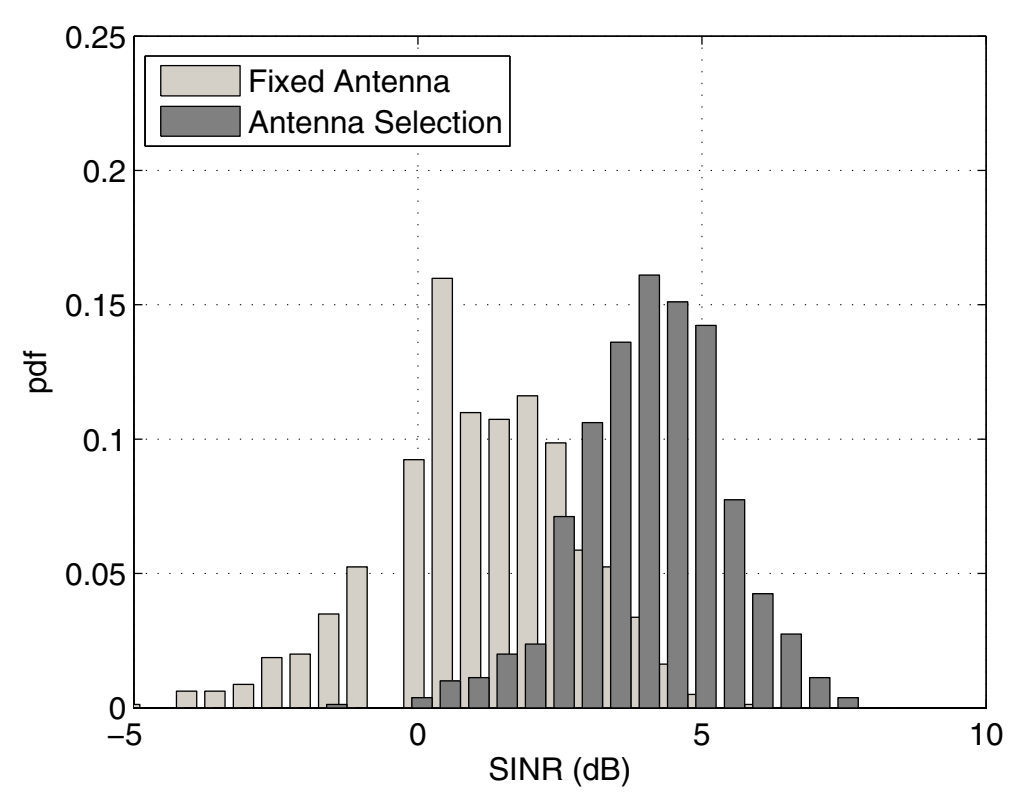

Figure 11 Empirical SINR distributions for Trial 3. 


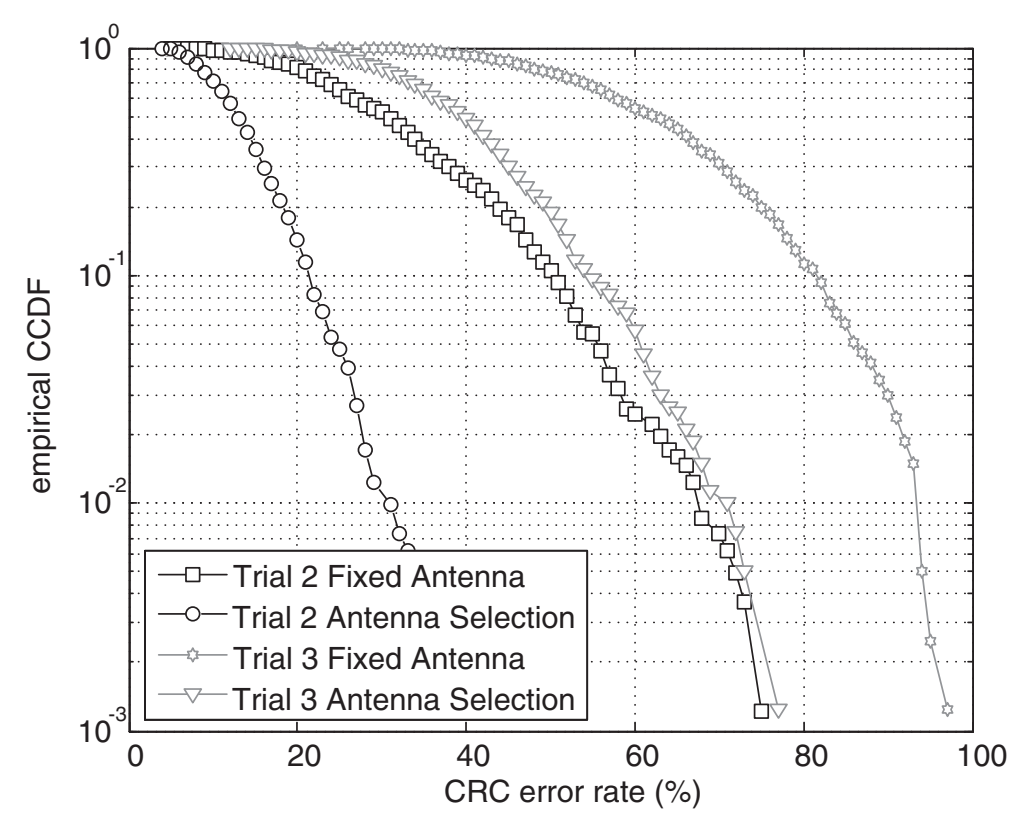

Figure 12 Empirical error rate CCDFs for interference trials.

channel quality variations are observed for realistic indoor channel models. Table 5 summarizes the average SINR improvements observed for different trials employing TGn models on channel emulation hardware.

Gains in the order of $1-2 \mathrm{~dB}$ are recorded in an interference-free case for an LTE femtocell access point selecting one out of four available antennas. However,

Table 5 Summary of results with channel estimation

\begin{tabular}{lccc}
\hline Trial & UE speed $(\mathbf{m} / \mathbf{s})$ & Training & $\boldsymbol{\Delta}$ SINR (dB) \\
\hline Trial 1, Channel B & 0.1 & $T=100$ & 1.26 \\
(no interference) & & & \\
& 0.5 & $T=100$ & 0.96 \\
& 0.5 & $T=20$ & 1.28 \\
& 1.0 & $T=20$ & 0.76 \\
& 2.0 & $T=20$ & 1.08 \\
Trial 1, Channel C & 0.1 & $T=100$ & 1.89 \\
(no interference) & & & \\
& 0.5 & $T=100$ & 1.11 \\
& 0.5 & $T=20$ & 1.57 \\
& 1.0 & $T=20$ & 0.88 \\
Trial 2 & 2.0 & $T=20$ & 1.88 \\
(moderate interference) & 0.25 & $T=100$ & 2.13 \\
Trial 3 & & & \\
(severe interference) & 0.25 & $T=100$ & 2.77 \\
\hline
\end{tabular}

no net gains are observed when considering the insertion loss of switching devices, so careful consideration has to be given to the choice of a low-loss switch. For instance, MEMS devices with losses of the order of tenths of $\mathrm{dB}$ are available, and the problems associated with longer switching delays can potentially be overcome in practical multiantenna femtocell base stations by carefully designing the training schedule in order to ensure that not all the antenna elements are switched at the same time. In any case, the diversity added by antenna selection may be beneficial in cases where the propagation channel remains static, reducing the probability of experiencing deep fades for prolonged times, as is demonstrated by an actual over-the-air experiment.

Finally, the benefits of antenna selection are more obvious when interference is the main limiting factor, as is expected to be in practice. In this case the insertion loss of the switches, which affects signal and interference equally, has limited effect on receiver SINR, making net gains easily achievable. Substantial improvements in terms of signal quality and average error rates are observed for both moderate and high interference cases.

These results establish antenna selection as a costeffective technique able to provide substantial performance gains in practical systems. Moreover, it can be easily combined with other physical layer techniques such as interference rejection combining in multiantenna base stations, paving the way for superior performance in future high-density femtocell access point deployments. 


\section{Endnote}

$$
\begin{aligned}
& \text { a } f(x)=o(g(x)) \Leftrightarrow \lim _{x \rightarrow 0} \frac{f(x)}{g(x)}=0 \text { or } \lim _{x \rightarrow \infty} \frac{f(x)}{g(x)}=0 \\
& \text { depending on context. }
\end{aligned}
$$

\section{Competing interests}

The authors declare that they have no competing interests.

\section{Acknowledgements}

The authors would like to thank their colleagues and Directors at the Toshiba Research Europe Ltd. for the support provided. Also, they would like to thank the Director of the Centre for Communications Research at the University of Bristol for providing access to their facilities and equipment, and Dr. Matthew Webb for his expert assistance with the channel emulation hardware.

Received: 24 October 2011 Accepted: 11 October 2012

Published: 2 November 2012

\section{References}

1. D Knisely, T Yoshizawa, F Favichia, Standardization of femtocells in 3GPP, IEEE Commun. Mag.47(9), 68-75 (2009)

2. D Lopez-Perez, A Valcarce, $G$ de la Roche, J Zhang, OFDMA femtocells: a roadmap on interference avoidance, IEEE Commun. Mag.47(9), 41-48 (2009)

3. JG Andrews, Interference cancellation for cellular systems: a contemporary overview, IEEE Commun. Mag.12(2), 19-29 (2005)

4. M Şahin, I Guvenc, MR Jeong, H Arslan, Handling CCl and ICI in OFDMA femtocell networks through frequency scheduling, IEEE Trans. Consumer Electron.55(4), 1936-1944 (2009)

5. TRen, R La, Downlink beamforming algorithms with inter-cell interference in cellular networks, IEEE Trans. Wirel. Commun.5(10), 2814-2823 (2006)

6. J Romero-Jerez, A Goldsmith, Receive antenna array strategies in fading and interference: an outage probability comparison, IEEE Trans. Wirel. Commun.7(3), 920-932 (2008)

7. M Hasna, MS Alouini, A Bastami, E Ebbini, Performance analysis of cellular mobile systems with successive co-channel interference cancellation, IEEE Trans. Wirel. Commun.2(1), 29-40 (2003)

8. H Claussen. Femtocell coverage optimization using switched multi-element antennas, in in Proc. IEEE Int. Conf. on Commun (Dresden, Germany, 2009, pp. 1-6

9. J Proakis, Digital Communications, 4th edn. McGraw-Hill, New York, 2001)

10. N-D Đào, Y Sun, WH Chin. Receive antenna selection techniques for femtocell uplink interference mitigation, in Proc. of Indoor Oudoor Femtocell workshop (IOFC), in conjunction with IEEE Conf. on Personal, Indoor, and Mobile Radio Commun. (PIMRC) ((Istanbul, Turkey, 2010), pp. 180-184

11. JP Coon, M Sandell, Combined bulk and per-tone transmit antenna selection in OFDM systems, IEEE Commun. Lett.14(5), 426-428 (2010)

12. A Molisch, M Win, M I M O systems with antenna selection, IEEE Microwave Mag.5(1), 46-56 (2004)

13. V Kristem, NB Mehta, AF Molisch, Optimal receive antenna selection in time-varying fading channels with practical training constraints, IEEE Trans. Commun.58(7), 2023-2034 (2010)

14. M Sandell. Analytical analysis of transmit diversity in WCDMA on fading multipath channels, in Proc. IEEE Conf. on Personal, Indoor, and Mobile Radio Commun (Osaka, Japan, 1999), pp. 1-5

15. L Zheng, D Tse, Diversity and multiplexing: a fundamental trade-off in multiple antenna channels, IEEE Trans. Inf. Theory.49(5), 1073-1096 (2003)

16. Z Wang, GB Giannakis, A simple general parameterization quantifying performance in fading channels, IEEE Trans. Commun.51(8), 1389-1398 (2003)

17. M Win, JH Winters, Analysis of hybrid selection/maximal-ratio combining in Rayleigh fading, IEEE Trans. Commun.47(12), 1773-1776 (1999)

18. MS Alouini, M Simon, An MGF-based performance analysis of generalized selection combining over Rayleigh fading channels, IEEE Trans. Commun.48(3), 401-415 (2000)

19. 3GPP Technical Specification, Evolved Universal Terrestrial Radio Access (E-UTRA); Base Station (BS) Radio Transmission and Reception (2009). [V8.6.0]
20. V Erceg, L Schumacher, P Kyritsi, et al., TGn Channel Models, IEEE 802.11 document 11-03/0940r4 (2004)

21. J Soler-Garrido, WH Chin. Performance of an LTE femtocell base station employing uplink antenna selection, in Proc. IEEE Wireless Adv. Conf, London, UK, 2011), pp. 224-229

doi:10.1186/1687-1499-2012-335

Cite this article as: Soler-Garrido et al:: Interference mitigation in an LTE femtocell base station using uplink antenna selection. EURASIP Journal on Wireless Communications and Networking 2012 2012:335.

\section{Submit your manuscript to a SpringerOpen ${ }^{\circ}$ journal and benefit from:}

- Convenient online submission

- Rigorous peer review

- Immediate publication on acceptance

- Open access: articles freely available online

- High visibility within the field

- Retaining the copyright to your article

Submit your next manuscript at $\boldsymbol{~ s p r i n g e r o p e n . c o m ~}$ 